\title{
Stadt und soziale Ungleichheit ${ }^{1}$
}

In der Stadt wird Gesellschaft zur räumlichen Struktur. Die gesellschaftliche Formation, die in der europäischen Stadt des 19. Jahrhunderts physische Gestalt gewonnen hatte, war die bürgerliche Gesellschaft. Damit sind zwei Merkmale für die europäische Stadt im 19. Jahrhundert bestimmend geworden: die Polarität von Öffentlichkeit und Privatheit und die Klassenspaltung. Hans Paul Bahrdt hat die Polarisierung einer öffentlichen und einer privaten Sphäre seiner Definition von Stadt zugrunde gelegt: »Eine Stadt ist eine Ansiedlung, in der das gesamte ... Leben die Tendenz zeigt, sich zu polarisieren, d.h. entweder im sozialen Aggregatszustand der Öffentlichkeit oder in dem der Privatheit stattzufinden «. ${ }^{2}$ Die Polarität öffentlicher und privater Räume ist das erste Merkmal, das die physische Gestalt der europäischen Stadt im 19. Jahrhundert geprägt hat. Die Gesellschaft des 19. Jahrhunderts war zudem eine Klassengesellschaft. Auch das hat die europäische Stadt geprägt: »Die Bourgeoisie hat mit allen anderen Nationen der Erde mehr Verwandtes als mit den Arbeitern, die dicht neben ihr wohnen. Die Arbeiter sprechen andre Dialekte, haben andre Ideen und Vorstellungen, andre Sitten und Sittenprinzipien, andere Religion und Politik als die Bourgeoisie. Es sind zwei ganz verschiedene Völker «. ${ }^{3}$ Und dementsprechend ist »die Stadt ... eigentümlich gebaut, so daß man jahrelang in ihr wohnen ... kann, ohne je in ein Arbeiterviertel oder nur mit Arbeitern in Berührung zu kommen « 4 , »... hinreichend, um vor den Augen der reichen Herren und Damen mit starkem Magen und schwachen Nerven das Elend und den Schmutz zu verbergen, die das ergänzende Moment zu ihrem Reichtum und Luxus bilden $«^{5}$. Die tiefe Spaltung zwischen Eigentümern von Produktionsmitteln und jenen, die nichts besitzen als ihre Arbeitskraft, ist das zweite Merkmal, das die räumliche Struktur der europäischen Stadt im 19. Jahrhundert geprägt hat.

Die Polarität von Öffentlichkeit und Privatheit hat ihre die Stadt prägende Kraft eingebüßt. Schon Bahrdt hatte auf die industriellen und tertiären Großbetriebe hingewiesen, die weder öffentlich noch privat seien. Besonders deutlich wird diese Ero-

1 Der Text beruht auf einem Vortrag gleichen Titels auf der Veranstaltung der AG Stadtleben und dem Forum für Politik und Kultur in memoriam Hartmut Häußermann am 8. Februar 2012 in Hannover. Ich danke Thomas Krämer-Badoni, Martin Kronauer und Helge Peters für kritische Hinweise und Reinhard Blomert für seine geduldige Redaktion, die den Text nicht nur sprachlich verbessert hat.

2 Bahrdt 1998, S. 83.

3 Engels 1970, S. 120.

4 Ebd., S. 70.

5 Ebd., S. 71. 
sion der Polarität von Öffentlichkeit und Privatheit mit der "Einhausung « (Gleichmann) des Marktes in den Galerien, später den Kaufhäusern und heute den großen Einkaufsmeilen, den Shopping Malls. Damit wird eine zentrale Funktion des öffentlichen Raums, seine Marktfunktion, in Räumen organisiert, die privatem Recht unterliegen. ${ }^{6}$ Im Zuge des 20. Jahrhunderts ist auch die soziale Spaltung der europäischen Stadt des 19. Jahrhunderts verschwunden. In der hohen Zeit des »rheinischen Kapitalismus " während der 1950er und 1960er Jahre war die gesellschaftliche Integration des Proletariats durch den fordistischen Dreiklang aus anhaltendem Wachstum, Ausbau des Sozialstaats und traditioneller Arbeitsteilung zwischen den Geschlechtern gelungen. Der von einer traditionellen Hausfrau geführte private Haushalt entlastete den Mann von allen außerberuflichen Verpflichtungen, sodass er sich ganz auf seinen Beruf konzentrieren konnte. Unbefristete Vollzeitbeschäftigung und steigende Reallöhne machten möglich, dass der berufstätige Mann eine Familie ernähren konnte. Und schließlich wurde ein sozialstaatliches Sicherheitsnetz geknüpft, das nicht nur Vorsorge trug für Notfälle wie Krankheit und vorübergehende Arbeitslosigkeit, sondern auch für ein materiell abgesichertes Leben im Alter. Nicht zuletzt haben eine sozial verantwortliche Stadterneuerungspolitik und ein beträchtliches Segment marktfern organisierter Wohnungsversorgung dazu beigetragen, dass krasse Erscheinungsformen sozialer Ungleichheit aus den deutschen Städten verschwunden sind.

Die europäische Stadt heute ist weit entfernt von dem, was Engels über das Manchester des 19. Jahrhunderts geschrieben hat. Aber ist es ausgeschlossen, dass die gespaltene Stadt des 19. im 21. Jahrhundert wiederkehrt? Diese Frage war das zentrale Thema der Forschungen von Hartmut Häußermann. Er hat die europäische Stadt eine Integrationsmaschine genannt, aber immer wieder danach gefragt, ob das Integrationsmodell der europäischen Stadt Bestand haben kann oder ob amerikanische Verhältnisse, wenn nicht gar die des 19. Jahrhunderts wieder bestimmend werden könnten. Das letzte Buch, an dem er vor seinem Tod zusammen mit Martin Kronauer gearbeitet hat, sollte dieses Thema behandeln. Häußermann hat damit die - neben der ökologischen - zentrale Frage nach der Zukunftsfähigkeit des europäischen Stadtmodells gestellt.

Es ist notwendig, diese Frage zu stellen, weil alle drei Säulen des fordistischen Integrationsmodells - Vollbeschäftigung, Sozialstaat und geschlechtspezifische Arbeitsteilung - brüchig geworden sind. Je weiter aber die Hochphase des Fordismus zurückliegt, in desto verklärterem Licht erscheint sie. Wenn in Diskussionen über den Wandel der Arbeitsmärkte, des Sozialstaats und der Lebensweisen der Fordismus nach dem Zweiten Weltkrieg implizit als normative Folie dient, vor deren Hintergrund die heutige Situation als Verlust interpretiert wird, dann bleibt dabei die Kritik ausgeblendet, die in den 1960er Jahren an eben dieser gesellschaftlichen Formation geübt wurde. Der Fordismus ist nicht das »verlorene Paradies «, in das man sich zurückwünschen sollte. Es war auch ein System entfremdeter Lohnarbeit, ungleicher Arbeitsteilung zwischen Mann und Frau, politischer Repression und des »Muffs unter den Talaren«. Dieses Gesicht des Fordismus wird allzu leicht verges-

6 Siebel 2006.

Leviathan, 40. Jg., 3/2012 
sen, wenn dem Prekariat das Normalarbeitsverhältnis, den Prozessen der Ausgrenzung die Mechanismen sozialstaatlicher Integration, den Alleinerziehenden und Alleinlebenden die Zwei-Generationen-Kleinfamilie und der gespaltenen die integrierte Stadt entgegengehalten werden. Wenn auch im Folgenden diese Kritik des Fordismus nicht weiter aufgegriffen wird, so ausschließlich, um das Thema einzuengen, nicht weil diese Kritik überholt wäre.

Der "Traum immerwährender Prosperität ${ }^{7}$ konnte nur kurz geträumt werden. Er hat die beiden goldenen Jahrzehnte nach dem Zweiten Weltkrieg nicht überdauert. Seit den 1970er Jahren hat die Integrationskraft der Arbeitsmärkte nachgelassen. Robert Castel ${ }^{8}$ spricht von der Herausbildung dreier Zonen: der Zone der Integration, der Zone der Vulnerabilität und der Zone der Ausgrenzung. Das Normalarbeitsverhältnis, charakterisiert durch unbefristete Beschäftigung, tariflich geregelte Arbeitsbedingungen und einen Lohn, der ausreicht, eine Familie zu ernähren und Vorsorge für Krankheit und Alter zu treffen, hat an Bedeutung verloren. Zwar sind noch nie so viele Menschen in Deutschland erwerbstätig gewesen wie gegenwärtig. Im Durchschnitt des Jahres 2010 waren 40,37 Millionen Personen in der BRD erwerbstätig. ${ }^{9}$ Aber die Zunahme der Beschäftigung beruht in erster Linie auf der Zunahme prekärer Beschäftigungsverhältnisse. 2008 war jeder Dritte ${ }^{10}$ "atypisch " beschäftigt, das heißt befristet, in Teilzeit-, Zeit- und Leiharbeit, in Minijobs und prekären Formen der Selbstständigkeit. Zehn Jahre früher waren es erst 18 Prozent aller Beschäftigten gewesen. Bestimmendes Merkmal dieser Formen der Erwerbstätigkeit ist Unsicherheit in einem doppelten Sinn: Die Arbeit selber ist unsicher, und ihre Erträge reichen nicht aus, um für Notfälle und Alter ausreichend Vorsorge tragen zu können. Es sind immer mehr Menschen in Arbeit, aber ihre Arbeit sichert immer weniger ihr Leben.

Unterhalb dieser Zone der Prekarität ist seit den 1970er Jahren ein Sockel der Langzeitarbeitslosigkeit entstanden, der fast unberührt von konjunkturellen Zyklen sich langsam verfestigt. Das wahre Ausmaß dieser Zone der Ausgrenzung wird verdeckt durch zirkuläre Karrieren in Fortbildungsmaßnahmen und Einrichtungen des zweiten Arbeitsmarkts. Betroffen sind in erster Linie Migranten, gewerblich Qualifizierte und Industriearbeiter, Unqualifizierte und Ältere, die im Ruhrgebiet und in nördlichen sowie östlichen Teilen der Republik leben. Längere Arbeitslosigkeit aber entfaltet eine eigenständige Dynamik: Mit jeder Phase ohne Arbeit steigt die Gefahr, erneut arbeitslos zu werden. Das Stigma des schwer Vermittelbaren heftet sich an die Betroffenen, sie werden nicht mehr zur Vorstellung eingeladen, am Ende geben sie die Suche auf. Ohne Arbeit zu sein beinhaltet nicht nur den Verlust ökonomischer Selbstständigkeit. Man verliert auch die Anerkennung, ein nützliches Mitglied der Gesellschaft zu sein. Es gibt immer weniger anerkannte Rollen außer-

7 Lutz 1984.

8 Castel 2000.

9 Statistisches Bundesamt 2011 a, S. 1.

1036 Prozent von in 2008 34,7 Millionen Erwerbstätigen; siehe Bartelheimer 2011, S. 386; vgl. auch Schmid 2012. 
halb der Erwerbsarbeit: Die Rolle der Hausfrau und Mutter ist abgewertet, die Rolle des Rentners wird immer später erst zugänglich, und Alter wird immer häufiger zu einer Phase relativer Armut.

Gleichzeitig sind die Filter schwächer geworden, die bislang verhindert hatten, dass das Arbeitsmarktschicksal auf das Wohnungsmarktschicksal eines Haushalts unvermittelt durchschlagen konnte. Zu diesen Filtern zählen das sozialstaatliche Sicherungsnetz, ein marktfernes Segment der Wohnungsversorgung und die privaten Mehrpersonenhaushalte.

Arbeitslosenversicherung, Krankenversicherung, Sozialhilfe und das Rentensystem hatten Haushalte mit zumindest einem Erwerbstätigen weitgehend gegen Notlagen geschützt. Zudem war seit Ende des Ersten Weltkriegs in Deutschland ein differenziertes System sozialpolitischer Vorkehrungen gegen Wohnungsnot und kurzfristigen Wohnungsverlust entwickelt worden. Das Mietrecht, der soziale Wohnungsbau, Wohngeld, die Übernahme der Wohnungskosten bei bedürftigen Haushalten und eine behutsame Stadterneuerungspolitik haben die Wohnungsversorgung unterer Einkommensschichten weitgehend von Marktmechanismen abgekoppelt. Dieses sozialstaatliche Netz der Wohnungsversorgung ist ein wesentlicher Grund für die Resistenz der deutschen Wohnungsmärkte gegen Hypothekenkrisen wie in den USA mit ihren fatalen Folgen für die betroffenen Haushalte. Deutschland hat im Vergleich zu anderen entwickelten westlichen Gesellschaften einen außerordentlich hohen Anteil an Mietwohnungen. 53 Prozent aller Haushalte wohnen hier zur Miete, ein weder im übrigen Europa noch in den USA auch nur annähernd erreichter Anteil. ${ }^{11}$ Das beruht nicht zuletzt darauf, dass auch Mieter rechtlich und finanziell weitgehend gegen plötzlichen Wohnungsverlust und Wohnungsnot gesichert sind. Deshalb sind hier anders als in den USA ökonomisch schwächere Haushalte nicht gezwungen, sich hoch riskant zu verschulden, um im Eigentumsmarkt eine scheinbar sichere Wohnsituation zu erreichen.

Das hat sich geändert. Die sozialstaatlichen Sicherungen reichen angesichts neuer Problemlagen nicht aus. Die Arbeitslosenversicherung war für vorübergehende Arbeitslosigkeit geschaffen, nicht für Langzeitarbeitslose. Diese sind daher nach Ende der vergleichsweise kurzen Versicherungszeiten auf Transferzahlungen angewiesen. Ähnlich gerichtete Veränderungen zeigen sich in der Stadt- und Wohnungspolitik. Wenn, wie neuerdings propagiert, Stadtpolitik sich darauf verlegt, die »Stärken zu stärken «, geht das bei anhaltend knappen Kassen notwendig zulasten der sozialen Aufgaben. Die von der SPD in den 1970er Jahren begonnene Politik, die Wohnungsversorgung wieder in den Markt zu überführen, hatte zur Folge, dass der soziale Wohnungsbau faktisch eingestellt wurde. Der Bestand an sozial gebundenen Wohnungen ist in Westdeutschland von ehemals 5 Mio. auf unter 1 Mio. geschrumpft, in Gesamtdeutschland auf unter 1,8 Mio., wobei nach Schätzungen jährlich 100.000 Wohnungen die Bindung verlieren. ${ }^{12}$

Ebenso problematisch wie dieser quantitative Rückgang der sozial gebundenen Bestände sind die damit verbundenen qualitativen Verschlechterungen. Die verblei-

11 ISI 2009, S. 3.

12 Siehe http://de.wikipedia.org/wiki/Sozialer_Wohnungsbau (Zugriff vom 05.07.2012).

Leviathan, 40. Jg., 3/2012 
benden sozial gebundenen Wohnungen konzentrieren sich in den (für Privatisierungen) unattraktiven Bauformen an unattraktiven peripheren Standorten. Außerdem verfolgt ein Teil der neuen Eigentümer hoch problematische Strategien: Nach einer Studie über Mehrfachverkäufe größerer Wohnungsbestände in den Jahren 1999-2008 haben nahezu alle Käufer die Mieten erhöht, aber die Bestandsinvestitionen spürbar gesenkt oder auf niedrigem Niveau gehalten. ${ }^{13}$ Und schließlich verlieren die Kommunen durch die Privatisierung kommunaler und gemeinnütziger Wohnungsbauträger ihre wichtigsten Partner einer sozial verantwortlichen Stadtpolitik. Die Mehrheit der Käufer zeigt keinerlei Interesse, sich an wohnungs- und stadtentwicklungspolitischen Maßnahmen der Kommune zu beteiligen. ${ }^{14}$ Vereinzelt betreiben Investoren sogar eine aus Sicht sozial verantwortlicher Stadtpolitik desaströse Politik, die geradezu darauf gerichtet ist, soziale Brennpunkte entstehen zu lassen. In seiner Studie über »Leben im Plattenbau « hat Carsten Keller ${ }^{15}$ beschrieben, wie Eigentümer größerer Wohnungsbestände die problembeladenen Haushalte in den für Privatisierung ungeeigneten Beständen konzentrieren und anschließend in diese Bestände nicht mehr investieren.

Auch der zweite Filter, der das Wohnungsmarktschicksal vom Arbeitsmarktschicksal abkoppelt, wird schwächer. Auf dem Arbeitsmarkt agiert das einzelne Individuum, auf dem Wohnungsmarkt aber der Haushalt. Anders als Einpersonenhaushalte verfügen Mehrpersonenhaushalte häufig über mehrere Erwerbspersonen, weshalb der Arbeitsplatzverlust eines Mitglieds weniger gravierende Folgen für die Mietzahlungsfähigkeit des Haushalts haben kann. Seit den 1960er Jahren ist aber die Zahl der Einpersonenhaushalte ständig gewachsen auf mittlerweile fast 40 Prozent aller Haushalte. ${ }^{16}$ Auch die Zahl der Alleinerziehenden hat zugenommen, und etwa die Hälfte von ihnen lebt in Armut. ${ }^{17}$ Mit der wachsenden Zahl von Einpersonenhaushalten und Alleinerziehenden wächst die Zahl der verwundbaren Haushalte.

Die Städte, insbesondere die Großstädte, sind die Orte, wo diese Entwicklungen zuallererst und in aller Schärfe sichtbar werden. Hier konzentrieren sich die Verwerfungen des ökonomischen Strukturwandels von der Industrie- zur Dienstleistungsgesellschaft. Deshalb ist hier auch die Arbeitslosigkeit höher als im Durchschnitt der BRD. Die vulnerablen Haushalte wohnen vor allem in den Großstädten und hier in den Innenstädten: 61 Prozent der Haushalte in den Innenstädten sind Einpersonenhaushalte; jeder Achte benötigt Hilfe zum Lebensunterhalt oder Grundsicherung, 13 Prozent sind SGB II-Empfänger. Die Zuwanderung ist von jeher auf die großen Städte gerichtet. Jeder fünfte Bewohner der Innenstädte besitzt nicht die deutsche Staatsbürgerschaft, ${ }^{18}$ zählt man die deutschen Staatsbürger mit Migrati-

13 BBSR 2011, S. 4.

14 Ebd.

15 Keller 2005.

16 Nach dem Statistischen Jahrbuch 2011 waren es 38,6 Prozent.

17 Bartelheimer 2011, S. 388.

18 BBSR 2009, S. 5. 
onshintergrund hinzu, so machen Zuwanderer in Stuttgart und Frankfurt bereits 40 Prozent der Bewohner aus, bei den Kindern sind es 60 Prozent. Migranten aber sind überdurchschnittlich häufig arbeitslos.

Die Zone der Integration, die Zone der Vulnerabilität und die Zone der Ausgrenzung formen in der Stadt ein Mosaik aus luxuriös aufgewerteten Quartieren, Zonen des Übergangs und Räumen der Ausgrenzung. Auf die beiden letzteren will ich etwas ausführlicher eingehen, weil die Zonen des Übergangs in ihrer Ambivalenz hoch wichtige Räume für die Stadtentwicklung sind; auf die Räume der Ausgrenzung, weil hier »Stadt « nicht nur der Ort ist, wo Gesellschaft erscheint, sondern wo die Stadt selber als Ursache gesellschaftlicher Phänomene erkennbar wird.

Die Zone des Übergangs ist in sich äußerst heterogen. Die Zahlen zu prekären Beschäftigungsverhältnissen und zu Einpersonenhaushalten lassen sich deshalb nicht umstandslos als Ausweitung dieser Zone interpretieren. Auch liefern Indikatoren zur Arbeitsmarktsituation und zum Familienstand für sich genommen keine gültige Beschreibung von Lebenssituationen. Die Zunahme der Einpersonenhaushalte hat sehr unterschiedliche Ursachen: den demographischen Wandel - die Menschen werden immer älter, weshalb es mehr Verwitwete - insbesondere Frauen gibt, nicht jede Witwe aber ist arm und isoliert; den Wandel der Lebensweise: es wird immer später geheiratet - nicht jeder Single aber ist einsam; die Expansion des Bildungswesens: sehr viel mehr junge Menschen sind in Ausbildung und Studium nicht jeder Student aber kann zum Prekariat gerechnet werden. Die Zahl der atypisch Beschäftigten ist gestiegen, aber das heißt nicht, dass die Zahl der unbefristeten Vollzeitbeschäftigten entsprechend zurückgegangen wäre. Die Zunahme der atypischen Beschäftigung beruht wesentlich auf der Zunahme der Frauenerwerbstätigkeit, und dabei handelt es sich zum großen Teil um Frauen, die mit einem vollzeitbeschäftigten Mann zusammenleben. ${ }^{19}$ Hinzu kommen junge Menschen, die sich während der Ausbildung etwas dazuverdienen. Auch die heute so gerne beschworene Kreativwirtschaft ist ein weites Feld für prekäre Beschäftigungsverhältnisse, nur dass ihre Angehörigen ihre objektiv prekäre Lage subjektiv zu einer gewollten Boheme verklären. Auch sie sammeln sich in den Zonen des Übergangs, aber da sie in der Regel über zwar geringes ökonomisches, aber umso höheres soziales und kulturelles Kapital verfügen, können sie als ein Ferment für die positive Entwicklung ihrer Wohn- und Arbeitsgebiete wirken. Die Zone des Übergangs ist also auch ein Raum urbaner Kreativität.

Ernest Burgess ${ }^{20}$ hat sie vor über 80 Jahren am Beispiel Chicagos beschrieben. Er meinte damit die Altbaugebiete am Rand der Innenstadt. Es seien City-Erwartungsgebiete, wo nicht mehr investiert wird in der Erwartung, dass der zentrale Geschäftsbezirk sich hierhin ausdehnen werde, was für die Zukunft ganz neue Verwertungschancen eröffnen könnte. Solange aber diese andere Zukunft noch nicht eingetreten ist, entsteht aus solchen Spekulationen ein heruntergewirtschaftetes Gebiet mit billigen Wohnungen und vielen leeren Räumen, geeignet, all das zu beherbergen, was in den geordneten Räumen der Stadt keinen Platz findet: die Enklaven der Armen,

19 Bartelheimer 2011, S. 388.

20 Burgess 1974.

Leviathan, 40. Jg., 3/2012 
der ethnischen Minderheiten, der Menschen in nichtfamilialen Lebensformen, vor allem aber der Menschen in Situationen des Übergangs. Das sind: junge Menschen zwischen Jugend und Erwachsensein, in Ausbildung oder Studium, in der Phase der Berufsorientierung, vor der Gründung einer Familie oder nach deren Scheitern, alte Menschen zwischen Autonomie und Abhängigkeit, Transnationale, die hier und ganz woanders leben, Migranten zwischen zwei Kulturen, Arbeitslose und Menschen in prekären Beschäftigungsverhältnissen.

Die Räume des Übergangs sind Räume hoher Fluktuation. Dementsprechend schwach sind die sozialen Kontrollen. Dies wiederum bedingt überdurchschnittliche Anteile an psychischen Erkrankungen, illegalen Aktivitäten und abweichenden Verhaltensweisen. Hier finden sich Rotlichtviertel und generell die Orte, an denen der brave Bürger seinen weniger reputierlichen Neigungen nachgeht. Es ist der Raum der Nachtseiten der Urbanität. Von diesem Raum ist in den Konzepten für die Innenstädte selten die Rede. Es sollte aber davon die Rede sein. Denn dieser Raum ist nicht nur ein Auffangbecken für alles, was in der geordneten Stadt nicht gern gesehen ist. Er ist ein notwendiger Raum der Stadt, ein Raum für unausgegorene Ideen, für Kreative und Existenzgründer, ein Raum der Vielfalt, des Unangepassten, der Überraschungen, der Begegnung mit dem Fremden, kurz: ein Raum urbaner Kultur.

Anders die Räume der Ausgrenzung. Sie sind nur schwer zu verorten. Ausgrenzung ist kein Zustand, und deshalb hat sie auch keinen bestimmten Ort in der Stadt. Von Ausgrenzung ist sinnvoll nur in zweierlei Hinsicht zu sprechen. Zum einen als relationaler Begriff: Von Ausgrenzung ist die Rede vor dem Hintergrund gelingender gesellschaftlicher Integration in den goldenen Zeiten des rheinischen Kapitalismus. Ausgrenzung meint die Vertreibung aus dem » fordistischen Paradies «. Zum anderen als dynamischer Begriff: Ausgrenzung ist ein Prozess, der Menschen oder auch städtische Räume an den Rand der Gesellschaft drängt, aber niemals über diesen Rand hinaus in ein Außerhalb der Stadt oder der Gesellschaft. Es gibt kein Außen der Gesellschaft. Simmel hat allenfalls den Bewohnern des Sirius den Status eines Außen der Gesellschaft zugestanden. ${ }^{21}$ Der Arme dagegen sei durchaus Teil der Gesellschaft, weil er als Adressat der Armenpflege in soziale Wechselbeziehungen eingebunden sei, allerdings nur als Objekt der Fürsorge und damit in sehr einseitige "Wechselbeziehungen ". ${ }^{22}$ Will man Ausgrenzungsprozesse verorten, muss man nach Stadtquartieren suchen, die »negative« Karrieren begünstigen. Solche Quartiere findet man an unattraktiven Standorten der Peripherie oder auch nur als Enklaven in der Zone des Übergangs, häufig handelt es sich nur um einzelne Straßenblocks oder Gebäude. In diesen Räumen können sich Kontext- oder Nachbarschaftseffekte ergeben, die eigenständige, zusätzliche Benachteiligungen zur Folge haben. Dort fungiert Stadt nicht als Ort, wo Ungleichheit erscheint, sondern als eine Ursache sozialer Ungleichheit. Dass solche Kontexteffekte bestehen, hat

21 Simmel 1992, S. 765.

22 Simmel 1993, S. 34. 
Andreas Farwick ${ }^{23}$ zum ersten Mal für Deutschland empirisch nachgewiesen am Beispiel von Stadtquartieren mit hohen Anteilen von Haushalten, die von Transferzahlungen leben. Wie sind diese Effekte zu beschreiben?

Münch ${ }^{24}$ definierte Ausgrenzung durch die Überlagerung von ökonomischer, politischer, sozialer und kultureller Marginalität. Kronauer fasst das zu drei Dimensionen versagter Teilhabe zusammen: »Marginalisierung am Arbeitsmarkt bis hin zum gänzlichen Ausschluss von Erwerbsarbeit; Einschränkung der sozialen Beziehungen bis hin zur Vereinzelung und sozialen Isolation; Ausschluss von Teilhabemöglichkeiten an gesellschaftlich anerkannten Lebenschancen und Lebensstandards «. ${ }^{25}$ In den Räumen der Ausgrenzung treten soziale, kulturelle und ökonomische Randständigkeit untereinander und mit einer degradierten Umwelt in Wechselwirkung. ${ }^{26}$ Diese Wechselwirkung kann Teufelskreiseffekte zur Folge haben, also eine besondere Dynamik von Ausgrenzungsprozessen auslösen. Damit wird die Stadt zu einem eigenständigen Faktor, der Ausgrenzung wenn nicht erzeugt, so doch verstärkt und dynamisiert. Die Stadt als eine Ursache von Ausgrenzungsprozessen aber wäre die härteste Verneinung des Versprechens der europäischen Stadt auf Integration.

Kontext- oder Nachbarschaftseffekte treffen vor allem jene, die weniger mobil und deshalb in erster Linie auf die Ressourcen des Quartiers angewiesen sind. Immobilität hat viele Gründe: Armut, Alter, Krankheit, die Angst vor Diskriminierung, Zeitzwänge, wie sie vor allem Alleinerziehende zu bewältigen haben, aber auch kulturelle Normen, die es beispielsweise Frauen verbieten, sich ohne männliche Begleitung in der Stadt zu bewegen. Kontexteffekte sind in vier Dimensionen zu vermuten. ${ }^{27}$

- Materiell: Der Zustand der Wohnungen und der öffentlichen Infrastruktur ist schlecht, die Versorgung mit Gütern des täglichen Bedarfs eingeschränkt, wichtige Dienstleistungen etwa in den Bereichen Bildung, Gesundheit, Beratung sind von minderer Qualität oder fehlen ganz. Es gibt fast keine Arbeitsplätze. In den Großsiedlungen, die in den späten 1960er bis in die 1980er Jahre im Umland der großen Städte errichtet worden sind, wächst zudem die Diskrepanz zwischen der gebauten Umwelt und der Lebenssituation der Bewohner. Diese Siedlungen waren auf das fordistische Lebensmodell von berufstätigem Mann und Hausfrau zugeschnitten. ${ }^{28}$ Dass sich außer Frauen, die mit Haushalt und Kindern beschäftigt waren, sonst noch jemand dort tagsüber aufhalten könnte, war nicht vorgesehen. In diese gerade für sie besonders ungeeigneten Bestände werden nun Arbeitslose, Alte, Alleinerziehende, Zuwanderer und andere Menschen, die

23 Farwick 2004.

24 Münch 1997.

25 Kronauer 2010, S. 145.

26 Häußermann et al. 2004.

27 Ebd., S. 24 f.

28 Kronauer 2010, S. 206 ff.

Leviathan, 40. Jg., 3/2012 
besonders auf die Ressourcen ihrer näheren Umgebung angewiesen sind, konzentriert.

- Sozial: Arme und Arbeitslose verfügen typischerweise über geringes soziales Kapital. Ihre sozialen Netze sind von geringer Reichweite und Leistungsfähigkeit. Sie umfassen in der Regel nur wenige Personen, sind sozial sehr homogen und verfügen nur über geringe Ressourcen. Arme können einander nicht mit Geld aushelfen, und Arbeitslose verfügen selten über Informationen zu Arbeitsmöglichkeiten, weil sie keinen Zugang zu Betrieben haben. ${ }^{29}$

- Symbolisch: Das Quartier hat ein schlechtes Image. Wer bei Bewerbungen eine solche Adresse angeben muss, hat von vornherein schlechtere Chancen, überhaupt eingeladen zu werden.

- Kulturell: In solchen Quartieren kann sich eine Kultur der Randständigkeit entwickeln. Jugendliche begegnen hier kaum Vorbildern, die die üblichen Wege der Integration über Schule, Ausbildung und Berufsarbeit erfolgreich beschritten haben. So kann die marginale Situation der Eltern vererbt werden.

Räume der Ausgrenzung entstehen in dem Maße, wie diese verschiedenen Dimensionen gesellschaftlicher Benachteiligung miteinander in Wechselwirkung treten und so Teufelskreiseffekte auslösen. Solche nach unten gerichteten Dynamiken können räumlich vermittelt sein, sie können durch Konflikte ausgelöst werden, und sie entstehen durch die Verschränkung objektiver Benachteiligung und negativer Selbstdefinition.

Es sind zwei räumlich vermittelte Prozesse, die eine negative Stadtteilkarriere auslösen können. Einmal Fahrstuhleffekte: Die Krise der Industriearbeitsmärkte hat sich insbesondere in den traditionellen Wohngebieten der Arbeiter niedergeschlagen. Hier ergaben sich Arbeitslosenquoten von 20 bis 40 Prozent. Wenn eine Zeche schließt, dann wird ein Großteil der Bewohnerschaft der zugeordneten Werkssiedlung arbeitslos. Die Siedlung und ihre Bewohner fahren gleichsam mit einem Fahrstuhl nach unten, ein Beispiel für die Stadt als Erscheinungsort gesellschaftlicher Prozesse.

Zum anderen können selektive Abwanderungen den Verfall eines Quartiers zur Folge haben. Wenn sich das Image eines Quartiers verschlechtert oder etwa durch Verkehrsausbau die Umweltsituation, dann zieht, wer kann, aus einem solchen Quartier fort. Mobilitätsfähig sind in erster Linie bessergestellte Haushalte der deutschen Mittel- und Oberschicht, aber auch integrationserfolgreiche Migranten. Die Fortzüge gerade dieser Haushalte lassen die Kaufkraft im Quartier sinken. In der Folge schränken private Anbieter ihre Güter- und Dienstleistungsangebote ein, die Banken werden zurückhaltend bei der Vergabe von Krediten, was wiederum Hauseigentümer veranlasst, nicht mehr ausreichend in ihre Bestände zu investieren, das Gebiet verkommt auch äußerlich, und wenn dann noch der Anteil der Kinder aus » bildungsfernen Schichten « in den örtlichen Schulen steigt, so ist das Anlass für die Abwanderung weiterer Haushalte, die sich Mobilität leisten können. Im Zuge eines Prozesses passiver Segregation konzentriert sich eine benachteiligte Bevölkerung in einem heruntergekommenen Gebiet mit mangelhafter Versorgung, einem 
schlechten Image und einer Kultur der Randständigkeit, wodurch sich die Kontexteffekte weiter verdichten.

Innerhalb der Räume des Übergangs kann - das ist der zweite Mechanismus eines circulus vitiosus der Ausgrenzung - eine Dynamik aggressiver gegenseitiger Abgrenzung in Gang kommen. Die Filtermechanismen auf den Wohnungsmärkten leiten die Zuwanderer gerade in diese gefährdeten Räume der Stadt, wo sie in Nachbarschaft zu den deutschen Verlierern des Strukturwandels geraten. Verlierer sind selten in der Lage oder auch nur willens, offen und tolerant auf Fremde zuzugehen, im Gegenteil, sie brauchen Sündenböcke - eine Rolle, für die sich die Fremden von jeher geeignet haben. Wenn solche erzwungenen Nachbarschaften zwischen einheimischen Verlierern und nicht integrierten Zuwanderern sich obendrein in einer städtischen Umwelt ergeben, die ihren Bewohnern tagtäglich vor Augen führt, dass sie am Rand der Stadtgesellschaft angekommen sind, dann ist es nicht verwunderlich, dass solche Räume nicht Orte gelingender Integration werden, sondern Orte konflikthafter gegenseitiger Abgrenzung.

Schließlich können sich objektive Lage und subjektive Reaktionen zu einem Syndrom der Randständigkeit verschränken, aus dem die Subjekte keinen Ausweg mehr erkennen. Das negative Image eines Viertels lässt das Selbstbild seiner Bewohner auf Dauer nicht unberührt. Wer um den schlechten Ruf seiner Wohngegend weiß und ihn täglich durch eine verfallende Umwelt und problematische Nachbarn bestätigt sieht, der hat es schwer, dagegen ein positives Selbstbild zu behaupten. Wer sich aber selbst als am Rande der Gesellschaft definiert, hat die Hoffnung aufgegeben. Die tägliche Erfahrung, am Rande der Gesellschaft zu leben, bleibt subjektiv nicht folgenlos. In solchen Quartieren kann sich die Überzeugung durchsetzen, von dieser Gesellschaft nichts erwarten zu können und deshalb ihr auch nichts schuldig zu sein. Das Resultat sind Apathie, Vandalismus und gelegentlich gewalttätige Ausbrüche.

Diese negative Wechselwirkung zwischen objektiver Lage und subjektiver Reaktion hat auch eine politische Dimension, die zur negativen Karriere eines Quartiers beiträgt. In den Quartieren der Ausgrenzung werden Menschen in sehr unterschiedlichen Lebenssituationen zusammengezwungen: Arme, kürzlich Zugewanderte, deutsche Arbeitslose, Alleinerziehende, vereinsamte alte Menschen. Ihre unterschiedlichen Interessen sind kaum politisch zu organisieren. Die Fragmentierung der Beherrschten aber ist - so zuletzt Boltanski ${ }^{30}$ - die effektivste Form der Sicherung von Herrschaft. Das wiederum verstärkt den Eindruck versteinerter Machtverhältnisse, an denen nichts zu ändern sei. Die Hoffnung, solche Orte könnten zu Orten besonderer Solidarität unter den Benachteiligten werden, ist illusionär. Das dichte Nebeneinander von Menschen in unterschiedlichen Situationen der Prekarität und dementsprechend mit unterschiedlichen Interessenlagen führt eher zu Konflikten oder zum Rückzug in die eigenen vier Wände als letztem Hort scheinbarer Sicherheit. Nach einer Studie von Podszuweit und Schütte ${ }^{31}$ summieren sich in St. Pauli Wahlenthaltung, fehlendes Wahlrecht und Stimmen für rechtsradikale Splitterparteien auf 70-80 Prozent. Das heißt, nur 20-30 Prozent der erwachsenen Bevölkerung

30 Boltanski 2010, S. 73.

31 Podszuweit, Schütte 1997, S. 32.

Leviathan, 40. Jg., 3/2012 
dieses Stadtteils sind in der Bürgerschaft vertreten. Da durch den Verkauf gemeinnütziger Wohnungsbestände diese Quartiere in einigen Städten auch die Wohnungsbaugesellschaften als Stellvertreter ihrer Interessen in der Kommunalpolitik verloren haben (s.o.), sind gerade jene Stadtteile, die am meisten der politischen Aufmerksamkeit bedürfen, am wenigsten in der Stadtpolitik präsent.

Der fordistische Dreiklang aus Normalarbeitsverhältnis, integrativer Sozial- und Stadtpolitik und geschlechtsspezifischer Arbeitsteilung ist verweht. Wachsende materielle Ungleichheit, zunehmende kulturelle Heterogenität, eine sich verbreiternde Zone des Prekariats, eine auf Wachstumsförderung orientierte Stadtpolitik und die Deregulierung der Wohnungsversorgung - diese Tendenzen zusammen formen ein Szenario scharfer sozialräumlicher Polarisierungen in den Städten. Auf der einen Seite drängen hochqualifizierte, einkommensstarke Haushalte mit nichtfamilialen Lebensweisen in die Kernstädte. Auf der anderen Seite konzentrieren sich Migranten und Menschen in prekären Lebensverhältnissen in den Städten. Und dort, wo Langzeitarbeitslosigkeit, schwache soziale Netze und Rückzug des Sozialstaats zusammentreffen, können Ausgrenzungsdynamiken in Gang gesetzt werden. Die von Häußermann gestellte Frage, ob sich die Klassenspaltung der Stadt des 19. Jahrhunderts in der Stadt des 21. Jahrhunderts wiederhole, kann damit allenfalls metaphorisch positiv beantwortet werden: Ja, es gibt Tendenzen in Richtung einer Spaltung der Stadtgesellschaft, die von ähnlicher Tiefe wie die der Stadt des 19. Jahrhunderts sein könnten, aber es ist doch in Erscheinungsformen, Ursachen und politischen Perspektiven eine gänzlich andere als die in Proletariat und besitzende Klassen.

Die Krise des fordistischen Reproduktionsmodells von männlichem Ernährer und Hausfrau könnte noch eine weitere Dimension sozialer Ungleichheit zur Folge haben, für die ebenfalls die Stadt als Ursache mitverantwortlich ist. Im traditionellen Modell geschlechtlicher Arbeitsteilung konnte der Mann sich ganz auf seinen Beruf konzentrieren, solange er über einen privaten Haushalt verfügte, geführt von einer Hausfrau, die ihn von allen außerberuflichen Verpflichtungen entlastete. Heute leben immer mehr Frauen berufs- und karriereorientiert und benötigen nun ihrerseits Entlastung von außerberuflichen Verpflichtungen: »A professional woman needs a wife " (Ray Pahl). Wenn immer mehr Menschen ein berufszentriertes Leben führen, die klassischen sozialen Voraussetzungen aber, um das zu können, fehlen, gibt es nur zwei Auswege aus diesem Dilemma: die radikale Reduktion außerberuflicher Verpflichtungen insbesondere durch den Verzicht auf Kinder - und das Leben in der Stadt. Moderne Dienstleistungsstädte sind Maschinen zur Entlastung von Arbeit und Verpflichtungen. Die Kinder, die Alten, die Kranken und die Sterbenden überlässt man den entsprechenden sozialen Einrichtungen, und noch für das intimste körperliche und psychische Bedürfnis findet man vielfältige Befriedigungsmöglichkeiten. Moderne Städte mit ihrer Überfülle an marktförmig und staatsförmig organisierten Güter- und Dienstleistungsangeboten sind zu begreifen als eine Form der Vergesellschaftung des privaten Haushalts. Sie sind damit die Voraussetzung für das berufszentrierte Leben gut verdienender Arbeitskräfte mit nichtfamilialen Lebensweisen. 
Aber die Vergesellschaftung der Haushaltungsarbeit hat negative Verteilungswirkungen. Der Zugang zu den Leistungen der Stadtmaschine ist für diejenigen kein Problem, die über die notwendigen Informationen, Qualifikationen und das Geld verfügen, um sich die entsprechenden Dienstleistungen und Güter zu verschaffen. In den großen Städten konzentrieren sich aber auch die prekär Beschäftigten, die Armen, die Alleinlebenden und die Alleinerziehenden. Sie sind besonders vulnerabel, sofern sie weder über Geld noch über einen zweiten Berufstätigen noch über die informellen Arbeitskapazitäten einer Hausfrau verfügen. Sie sind daher besonders angewiesen auf die marktförmig oder staatsförmig organisierten Güterangebote und Dienstleistungen der Stadt. Aber sie gewinnen nur schwer oder gar nicht Zugang zu den Leistungen der Stadtmaschine. Möglicherweise könnte daraus eine weitere Dimension der Polarisierung der Stadt entstehen zwischen denen, die sich die Stadtmaschine leisten können, und jenen, die aus Mangel an Geld, Informationen und Rechten keinen Zugang dazu finden. Und diese Spaltung innerhalb des Reproduktionsbereichs wird sich in dem Maße vertiefen, wie die Kommunen sich aus der Organisation entsprechender Güter- und Dienstleistungsangebote zurückziehen und etwa die Betreuung von Alten, Kranken und Sterbenden privatwirtschaftlich organisierten Betrieben überlassen.

Noch eine weitere und sehr viel grundsätzlichere negative Folgewirkung der Vergesellschaftung der Hausarbeit ist zu erwähnen. Die Rolle der Hausfrau kann vergesellschaftet werden, aber nicht die der Mutter. Vieles von dem, was alte Menschen brauchen, ist marktförmig oder staatsförmig organisierbar, aber das Wichtigste, was Kinder wie alte Menschen vor allem anderen benötigen, nicht: Achtung, Vertrauen und Liebe. Das sind Qualitäten menschlicher Beziehungen, die nicht professionell und gegen Geld verfügbar gemacht werden können. Sie bleiben gebunden an die informellen Systeme von Verwandtschaft, Freundschaft und Nachbarschaft. Die Folgen dieser anthropologischen Schranke der Vergesellschaftung von Leistungen, die als Reproduktionsarbeit vormals in Familienhaushalten in unbezahlter Arbeit vornehmlich von den Frauen erbracht wurden, werden alle mehr oder weniger unterschiedslos treffen, eben weil es darauf keine Antwort durch Markt und Staat gibt. ${ }^{32}$ Aber eine Lösung wäre nur zu finden, wenn eine ganz andere, grundlegende Ungleichheit überwunden würde: die ungleiche Arbeitsteilung zwischen Mann und Frau. Moderne Dienstleistungsstädte sind als Orte der Vergesellschaftung der Reproduktionsarbeit die bevorzugten Lebensorte der Menschen mit nichtfamilialen Lebensweisen. Sie werden daher auch die Orte sein, in denen sich diese Form von sozialer »Verarmung « konzentriert.

\section{Literatur}

Bahrdt, Hans Paul 1998 [1961]. Die moderne Großstadt. Opladen: Leske + Budrich.

Bartelheimer, Peter 2011. »Unsichere Erwerbsbeteiligung und Prekarität «, in WSI-Mitteilungen 8, S. 386-393.

32 Häußermann, Siebel 1995, S. 201 ff.

Leviathan, 40. Jg., 3/2012 
BBSR (Bundesinstitut für Bau-, Stadt- und Raumforschung) 2009. »Großstädtisches Flair gesucht? Oder wer wohnt in der Innenstadt? ", in Informationen aus der Forschung des BBSR 5, S. 5-6.

BBSR 2011. »Mehrfachverkäufe von Mietwohnungsbeständen - Forschungen Heft 146 «, in Informationen aus der Forschung des BBSR 2, S. 4.

Boltanski, Luc 2010. Soziologie und Sozialkritik. Frankfurt a. M.: Suhrkamp.

Burgess, Ernest W. 1974 [1925]. »The growth of the city «, in The city, hrsg. v. Park, Robert E.; Burgess, Ernest W.; McKenzie, Roderick D., S. 47-62. Chicago: University of Chicago Press.

Castel, Robert 2000. Die Metamorphosen der sozialen Frage. Eine Chronik der Lohnarbeit. Konstanz: UVK

Engels, Friedrich 1970 [1845]. Über die Umwelt der arbeitenden Klassen. Aus den Schriften von Friedrich Engels, ausgewählt von Günter Hillmann. Bauwelt Fundamente, Bd. 27. Gütersloh: Bertelsmann Fachverlag.

Farwick, Andreas 2004. "Segregierte Armut: Zum Einfluß städtischer Wohnquartiere auf die Dauer von Armutslagen ", in An den Rändern der Städte, hrsg. v. Häußermann, Hartmut; Kronauer, Martin; Siebel, Walter, S. 286-314. Frankfurt a. M.: edition suhrkamp.

Häußermann, Hartmut; Kronauer, Martin; Siebel, Walter 2004. »Einleitung «, in An den Rändern der Städte, hrsg. v. Häußermann, Hartmut; Kronauer, Martin; Siebel, Walter, S. 7-40. Frankfurt a. M.: edition suhrkamp.

Häußermann, Hartmut; Siebel, Walter 1995. Dienstleistungsgesellschaften. Frankfurt a. M.: edition suhrkamp.

ISI (Informationsdienst Soziale Indikatoren) 2009. »Wohnen in Deutschland: Teuer, komfortabel und meist zur Miete", in Informationsdienst Soziale Indikatoren 41, S. 1-7.

Keller, Carsten 2005. Leben im Plattenbau. Zur Dynamik sozialer Ausgrenzung. Frankfurt a. M.: Campus.

Kronauer, Martin 2010. Exklusion. 2., aktualisierte und erweiterte Auflage. Frankfurt a. M.: Campus.

Lutz, Burkart 1984. Der kurze Traum immerwährender Prosperität. Frankfurt a. M.: Campus.

Münch, Richard 1997. »Elemente einer Theorie der Integration moderner Gesellschaften «, in Was hält die Gesellschaft zusammen?, hrsg. v. Heitmeyer, Wilhelm, S. 66-112. Frankfurt a. M.: edition suhrkamp.

Podszuweit, Ulrich; Schütte, Wolfgang 1997. Sozialatlas Hamburg. Hamburg: Fachhochschule Hamburg.

Schmid, Günther 2012. »Arbeit versichern «, in Leviathan 40, 2, S. 248-270.

Siebel, Walter 2006. "Zum Wandel des öffentlichen Raums - das Beispiel Shopping-Mall «, in Stadt und Kommunikation in bundesrepublikanischen Umbruchszeiten, hrsg. v. Saldern, Adelheid von, S. 67-82. Wiesbaden: Frans Steiner Verlag.

Simmel, Georg 1992 [1908]. »Exkurs über den Fremden «, in Georg Simmel: Gesamtausgabe, Bd. 11, hrsg. v. Rammstedt, Ottheim, S. 764-771. Frankfurt a. M.: Suhrkamp.

Simmel, Georg 1993 [1908]. "Zur Soziologie der Armut ", in Georg Simmel: Gesamtausgabe, Bd. 8, hrsg. v. Rammstedt, Ottheim, S. 24-57. Frankfurt a. M.: Suhrkamp.

Statistisches Bundesamt Deutschland 2011 a. Zahl der Erwerbstätigen erreicht im Jahr 2010 neuen Höchststand. Pressemitteilung Nr. 001 vom 03.01.2011. Wiesbaden.

Statistisches Bundesamt Deutschland 2011 b: Haushalte nach Haushaltsgröße. Wiesbaden. https://www.destatis.de/DE/ZahlenFakten/GesellschaftStaat/Bevoelkerung/HaushalteFamilien/Tabellen/Haushaltsgroesse.html (Zugriff vom 05.07.2012). 
Zusammenfassung: Mit dem Ende des Fordismus werden auch die Filter schwächer, die lange Zeit verhindert haben, dass das Arbeitsmarktschicksal eines Haushalts auf sein Wohnungsmarktschicksal und damit auf die sozial-räumliche Struktur der Stadt unmittelbar durchschlagen konnte: sozialstaatliche Sicherungen, ein beträchtliches Segment marktfern organisierter Wohnungsversorgung und der Mehrpersonenhaushalt. Damit gewinnt die soziale Frage in den Städten wieder an Brisanz. Es entsteht ein Mosaik aus luxuriös aufgewerteten Quartieren, Zonen des Übergangs und Räumen der Ausgrenzung. Auf die beiden letzteren Quartierstypen wird ausführlicher eingegangen. Die Zonen des Übergangs bilden Nischen für die Schwächeren, aber auch für die Kreativen. Die Räume der Ausgrenzung machen sichtbar, dass die Stadt nicht nur als Erscheinungsort sozialer Ungleichheit fungiert, sondern auch als eine ihrer Ursachen.

Stichworte: Ende des Fordismus, Stadtentwicklung, Stadtstruktur, Ausgrenzung

\section{City and social inequality}

Summary: In the golden decades of capitalism after World War Two, social security, housing policies and family households effectively prevented labour market careers directly determining the career of a household on the housing market. With the end of Fordism these filter mechanisms have been weakened. The question of social inequality has thus gained relevance in city development. Cities have become mosaics composed of luxury quarters, zones of transition and spaces of exclusion. The zones of transition are characterised by highly heterogeneous social structures and ambivalent functions as niches for the poor and for the creative. The spaces of exclusion clearly illustrate that the city is not only a place of social inequality, it is one of its causes.

Keywords: Fordism, city development, urban structure, exclusion

\section{Autor}

Prof. Dr. Walter Siebel

Institut für Soziologie

Carl von Ossietzky Universität

26111 Oldenburg

Leviathan, 40. Jg., 3/2012 https://doi.org/10.5771/0340-0425-2012-3-462 